\title{
Formulation d'une poudre d'alumine et formation par compaction grande vitesse : influence sur les propriétés du comprimé
}

\author{
David Souriou ${ }^{1, a}$, Patrice Goeuriot ${ }^{1}$, Olivier Bonnefoy ${ }^{1}$, Gérard Thomas $^{1}$ \\ ET FLORENCE DORÉ2 \\ 1 École nationale supérieure des mines de Saint-Étienne, UMR CNRS 5146 et 5148, 158 cours Fauriel, \\ 42023 Saint-Étienne Cedex 2, France \\ 2 CETIM, 7 rue de la Presse, BP 802, 42952 Saint-Étienne Cedex 09, France
}

Reçu le 3 octobre 2008, accepté le 4 novembre 2008

\begin{abstract}
Résumé - La compression de poudre céramique nécessite l'incorporation de molécules organiques assurant la cohésion des grains entre eux. Comprimer des poudres céramiques formulées revient à leur fournir une certaine énergie. En fonction de la formulation de la poudre, on peut mesurer l'énergie stockée par unité de masse, caractéristique de la poudre. Récemment, une technique employée dans la métallurgie des poudres, la Compaction Grande Vitesse (CGV), a été utilisée pour la mise en forme d'objets céramiques. Cette méthode apporte des particularités au niveau des propriétés physiques des comprimés, notamment en terme d'homogénéité par rapport au pressage uniaxial dit conventionnel. Des comprimés issus des deux méthodes de mise en forme, et élaborés à partir de poudres formulées différemment, seront comparés en terme de comportement à la compression et via l'utilisation d'un modèle mathématique.
\end{abstract}

Mots clés : Compression / alumine / formulation / énergie / modèle

\begin{abstract}
Formulation of an alumina powder and high velocity compaction forming: influence on compact properties. The compression of ceramic powders requires the addition of organic materials to insure tablet cohesion. For the compaction of formulated ceramic powders it is necessary to supply them energy. As a function of the formulation of ceramic powders, one can measure the specific energy acquired during the tablet compression. Recently, a technique used in powder metallurgy, High Velocity Compaction (HVC), has been studied to form ceramic compacts. This method brings special physical properties to tablets in terms of homogeneity compared to conventional uniaxial pressing. The properties of tablets prepared by the two processes are compared and discussed through a phenomenological model.
\end{abstract}

Key words: Compression / alumina / formulation / high velocity compaction / model

\section{Introduction}

Le pressage uniaxial est une méthode de mise en forme classique dans le domaine des céramiques. Le but est l'obtention d'un comprimé cohésif et le plus dense possible. La cohésion est rarement assurée par les seules forces de surfaces entre les grains céramiques. C'est pourquoi des molécules organiques faisant office de « colle » entre les grains sont ajoutées. Suivant la formulation de cette « colle », le comportement de la poudre à la compression et les propriétés physiques du comprimé peuvent varier, notamment la densité à vert et la résistance mécanique.

\footnotetext{
a Auteur pour correspondance :

souriou.david@wanadoo.fr
}

Une nouvelle technique de mise en forme de poudres, la Compression Grande Vitesse (CGV), a été utilisée pour la mise en forme d'objets métalliques et céramiques. L'appareillage CGV est une presse HYDROPULSOR (appareillage installé au CETIM, Saint-Étienne). Cette technique est similaire au pressage uniaxial mais consiste à accélérer d'une certaine hauteur un bélier qui va frapper le poinçon supérieur de la matrice et provoquer une augmentation spontanée de la pression en un temps très court par rapport au pressage conventionnel (temps inférieur à la milliseconde). Les premières études ont montré que des pressions élevées (supérieures à $1 \mathrm{GPa}$ ) peuvent être atteintes et que les pièces obtenues sont plus homogènes [1].

L'objectif de l'étude est dans un premier temps de bien comprendre l'influence que la formulation d'une poudre a 
Tableau 1. Formulations des poudres d'alumine SM8 et B15 et nomenclatures.

\begin{tabular}{ccccccc}
\hline & \multicolumn{2}{c}{ PEG 4000} & $2 / 3 \mathrm{PVA}-1 / 3 \mathrm{PEG} 1500$ & \multicolumn{2}{c}{ 1/3PVA-2/3PEG1500 } \\
\hline SM8 (diamètre 0,4 $\mu \mathrm{m})$ & $2 \%$ & $4 \%$ & $2 \%$ & $4 \%$ & $2 \%$ & $4 \%$ \\
Nomenclature & SM8-2P & SM8-4P & SM8-2L/P & SM8-4L/P & SM8-2P/L & SM8-4P/L \\
B15 (diamètre 0,15 $\mu \mathrm{m})$ & $2 \%$ & $4 \%$ & $2 \%$ & $4 \%$ & - & - \\
Nomenclature & B15-2P & B15-4P & B15-2L/P & B15-4L/P & - & - \\
\hline
\end{tabular}

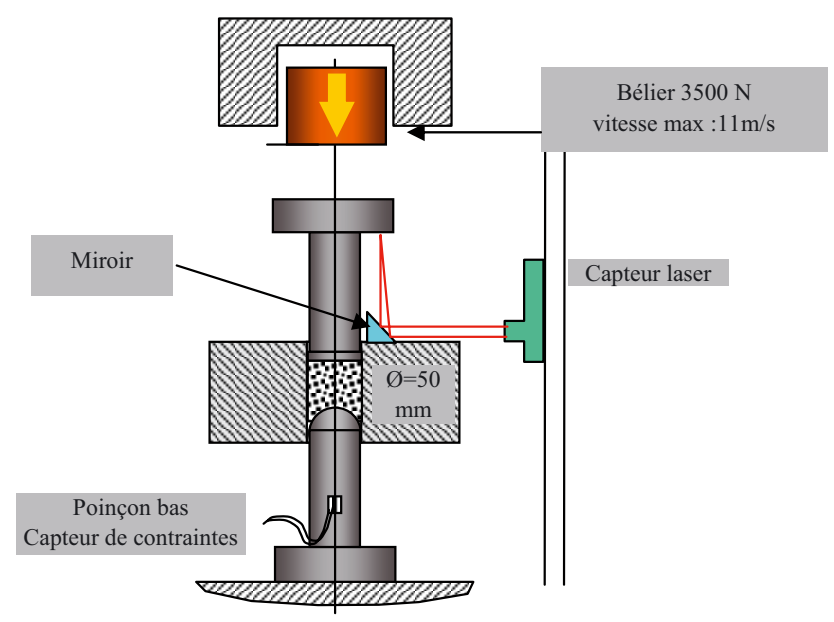

Fig. 1. Schéma du principe CGV.

sur la compression et les propriétés du comprimé puis comparer via un modèle mathématique le comportement à la compression d'un lit de poudre soumis à chacune des méthodes de mise en forme.

\section{Méthodes expérimentales}

Différentes poudres ont été élaborées par la société Baikowski afin de tester l'influence de la taille des cristallites et des formulations organiques sur les propriétés des comprimés. Deux types d'alumines sont utilisés : l'alumine SM8 de taille de grain de 0,4 $\mu \mathrm{m}$ et l'alumine B15, plus fine, de taille de grain de 0,15 $\mu \mathrm{m}$. L'alumine est choisie pour ses propriétés physiques (dureté) et rhéologiques (pas de déformation de type visqueux à température ambiante). Le choix de la formulation des poudres est fait sur la base d'un plastifiant pur (PEG 4000) et de mélanges liant-plastifiant (PVA/PEG 1500). Ces formulations ont été choisies en raison de leur emploi courant dans l'industrie. Le tableau 1 résume les différentes nomenclatures données aux 10 poudres.

Le pourcentage massique de matière organique est calculé d'après la masse d'alumine. La lettre $\mathrm{P}$ fait référence aux molécules plastifiantes, la lettre $\mathrm{L}$ fait référence aux molécules liantes. Dans les couples de lettre L et P, la lettre L en premier signifie qu'il y a une quantité dominante de liant et la lettre $\mathrm{P}$ en premier signifie que la quantité de plastifiant est dominante.

L'appareillage CGV est décrit dans la figure 1.

L'énergie de compaction est donnée par la chute d'un bélier de $350 \mathrm{~kg}$, accéléré par pression hydrolique à partir d'une hauteur maximum de $16 \mathrm{~mm}$ par rapport au

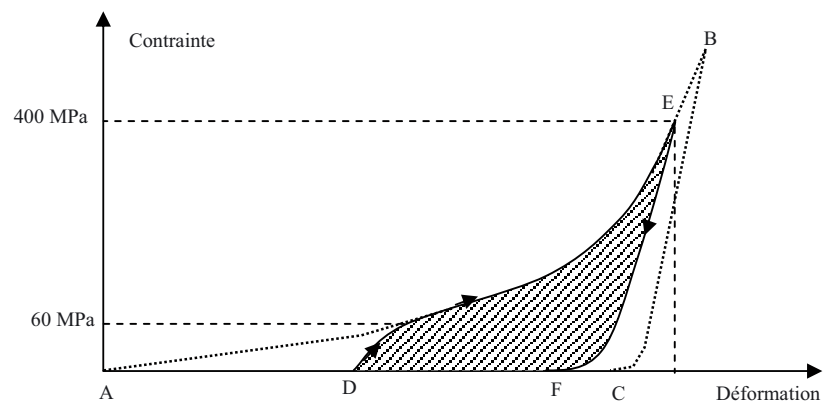

Fig. 2. Représentation des zones de travail de compression.

poinçon supérieur. De plus, cette presse est capable de frapper jusqu'à 5 coups par seconde tout en contrôlant l'énergie délivrée à chaque frappe. Les comprimés sont élaborés dans une matrice carbure de $50 \mathrm{~mm}$ de diamètre.

Un capteur de contrainte est placé sous le poinçon inférieur. Le déplacement du poinçon supérieur peut être connu via un capteur laser réfléchi par un miroir. Il est alors possible de tracer les courbes contraintes en fonction du déplacement et d'avoir accès aux informations utiles.

L'introduction de $70 \mathrm{~g}$ de poudre d'alumine dans la matrice laisse, après impact, un espace suffisant entre le poinçon supérieur et le miroir (le miroir n'est pas endommagé après l'essai). Toutefois, la limite de détection du déplacement par le capteur laser est de $10 \mathrm{~mm}$ (après étalonnage) et pour $70 \mathrm{~g}$ de poudre introduite, le déplacement du poinçon au cours de l'impact est supérieur à cette limite. C'est pourquoi une précompression de $60 \mathrm{MPa}$ de la poudre est effectuée avant chaque impact, de manière à tasser la poudre et détecter un déplacement.

Les compressions conventionnelles sont réalisées à l'aide d'une presse de traction-compression INSTRON, dans une matrice carbure de $20 \mathrm{~mm}$ de diamètre. Afin de pouvoir faire une comparaison entre mise en forme CGV et conventionnelle, la poudre est précomprimée à $60 \mathrm{MPa}$ puis comprimée à $400 \mathrm{MPa}$.

Les énergies ont été déterminées par le calcul de l'aire des courbes de compression. Elles correspondent au travail fourni pour comprimer les poudres soit :

$$
\mathrm{d} W=F \mathrm{~d} l .
$$

Les courbes de compressibilité obtenues (contraintedéplacement) permettent d'avoir accès à la mesure d'énergie stockée par la poudre au cours de la seconde compression qui est la différence de l'aire totale de la courbe enregistrée après la pré-compression moins celle du retour élastique de l'ensemble poudre-matrice à partir de $400 \mathrm{MPa}$ (aire hachurée DEF de la Fig. 2). 
Lors d'un essai de compression, la poudre mais également l'ensemble poinçon et matrice se déforment sous l'effet de la pression. Comme l'ensemble matrice et poinçon se déforme élastiquement, il est possible de retrancher leur comportement à la courbe globale de manière à se retrouver avec le comportement de la poudre seule. Il est ainsi possible de faire des comparaisons entre différents essais de compression qui n'ont pas été réalisés avec la même matrice de mise en forme.

Les courbes de compression font l'objet de modèles mathématiques exprimant la variation volumique des compacts en fonction de la pression appliquée. Ces modèles sont classiquement celui de Walker et de Maxwell, mais des modèles plus récents tels que celui de Sonnergaard et Heckel-Gonthier rendent mieux compte des phénomènes de fragmentation qui ont lieu lors de la compression de poudres céramiques. Le modèle de Lamy, un modèle généraliste très récent, considère la compression des poudres comme une réaction quasi-chimique [2]. Ce modèle repose sur l'équation suivante :

$$
\begin{aligned}
\frac{\varepsilon}{1-\varepsilon}= & \frac{\varepsilon_{0}}{1-\varepsilon_{0}}\left[\frac{\varphi}{k_{\mathrm{f}}+k_{\mathrm{CN}}}\left(k_{\mathrm{CN}}+\frac{k_{\mathrm{f}} \psi}{\psi-k_{\mathrm{f}}-k_{\mathrm{CN}}}\right)\right. \\
& \left.\times \mathrm{e}^{-\left(k_{\mathrm{f}}+k_{\mathrm{CN}}\right) P}+\left(1-\varphi-\frac{k_{\mathrm{f}} \varphi}{\psi-k_{\mathrm{f}}-k_{\mathrm{CN}}}\right) \mathrm{e}^{-\psi P}\right]
\end{aligned}
$$

avec :

- $\varepsilon$ (sans unité) porosité de l'empilement granulaire à la pression $P, \varepsilon_{0}$ : porosité initiale incluant la porosité intraparticulaire initiale $\left(\varepsilon_{i 0}\right)$ et de la porosité interparticulaire initiale $\left(\varepsilon_{e 0}\right)$.

- $P$ (en $\mathrm{MPa}$ ) est la pression de mise en forme variant au cours de l'essai de compression.

$-\varphi$ (sans unité) coefficient de «porosité »; défini par $\frac{\varepsilon_{i 0}}{\varepsilon_{i 0}+\varepsilon_{e 0}}$.

- $k_{\mathrm{f}}\left(\right.$ en $\left.\mathrm{MPa}^{-1}\right)$ coefficient de fragmentation; une valeur faible (typiquement inférieure à $10^{-4} \mathrm{MPa}^{-1}$ ) correspond à des grains cassant difficilement.

- $k_{\mathrm{CN}}\left(\right.$ en $\left.\mathrm{MPa}^{-1}\right)$ est le coefficient de déformation plastique; il représente la « vitesse de collage » des grains en cas de déformation plastique (une valeur plus faible correspond à des grains plus durs).

- $\psi\left(\mathrm{en}_{\mathrm{MPa}}{ }^{-1}\right)$ coefficient de réorganisation traduisant l'aptitude du système granulaire à se réarranger après un tassement, une fragmentation ou une déformation plastique d'un fragment de particule.

Les différents coefficients peuvent donner des informations sur le comportement de la poudre sous l'effet de la pression. Les coefficients $\varphi, k_{\mathrm{f}}, k_{\mathrm{CN}}$ et $\psi$ sont déterminés en utilisant la méthode des moindres carrés pour s'approcher au mieux des données expérimentales $\varepsilon=f(P)$. Ce modèle n'est valable que sur la compression de la poudre seule, d'où la nécessité de retrancher le comportement de la matrice. Les différents coefficients peuvent donner des informations sur le comportement de la poudre sous l'effet de la pression.

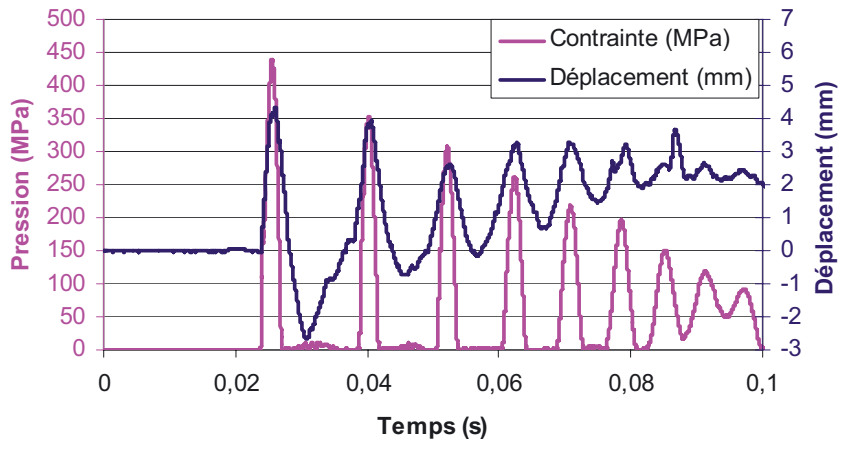

Fig. 3. Représentation des pics de contrainte et de déplacement d'un essai CGV.

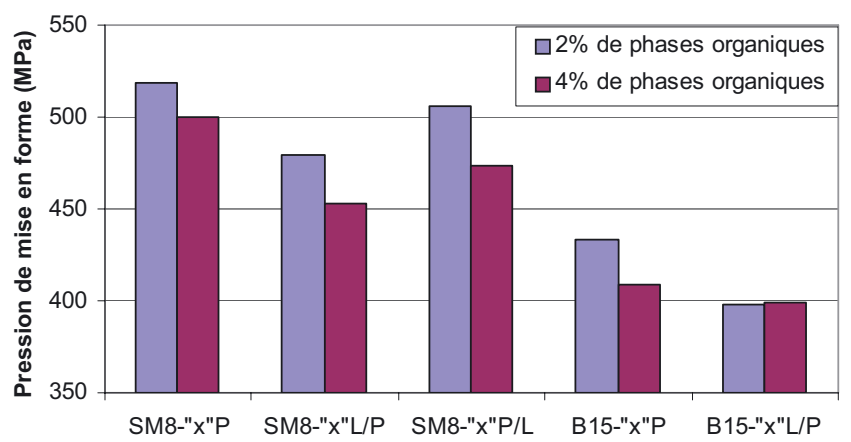

Fig. 4. Pression de mise en forme obtenue pour chacune des poudres d'alumine testées en CGV.

$\frac{\varepsilon}{1-\varepsilon}$ est déterminable expérimentalement et varie en fonction de la pression tandis que l'autre partie de l'équation correspond à une partie théorique où les valeurs des coefficients sont fixés arbitrairement dans des cellules isolées. On peut alors demander au logiciel de minimiser les différences entre l'expérimental et le théorique pour chaque valeur de la pression enregistrée au cours de l'essai en faisant varier les valeurs des cellules correspondant aux coefficients.

\section{Expériences}

\subsection{Mise en forme des comprimés}

Les comprimés CGV sont mis en forme avec une énergie d'impact de $2950 \mathrm{~J}$ ce qui correspond à une hauteur d'accélération de $21 \mathrm{~mm}$. Une mise en forme par impact comprend différents pics de pression décroissants au cours du temps. À titre d'exemple, la figure 3 montre les pics de pression et de déplacement obtenus lors de la mise en forme par impact de la poudre SM8-4L/P.

Seul le premier pic sera considéré par la suite comme le pic de contrainte principal, cette valeur correspondant à la pression de mise en forme. La montée et la descente en pression se fait dans un temps relativement court et on constate que les pics de contrainte et de déplacement sont en phase les uns avec les autres. La figure 4 regroupe la pression mesurée (soit le pic de contrainte principal) 

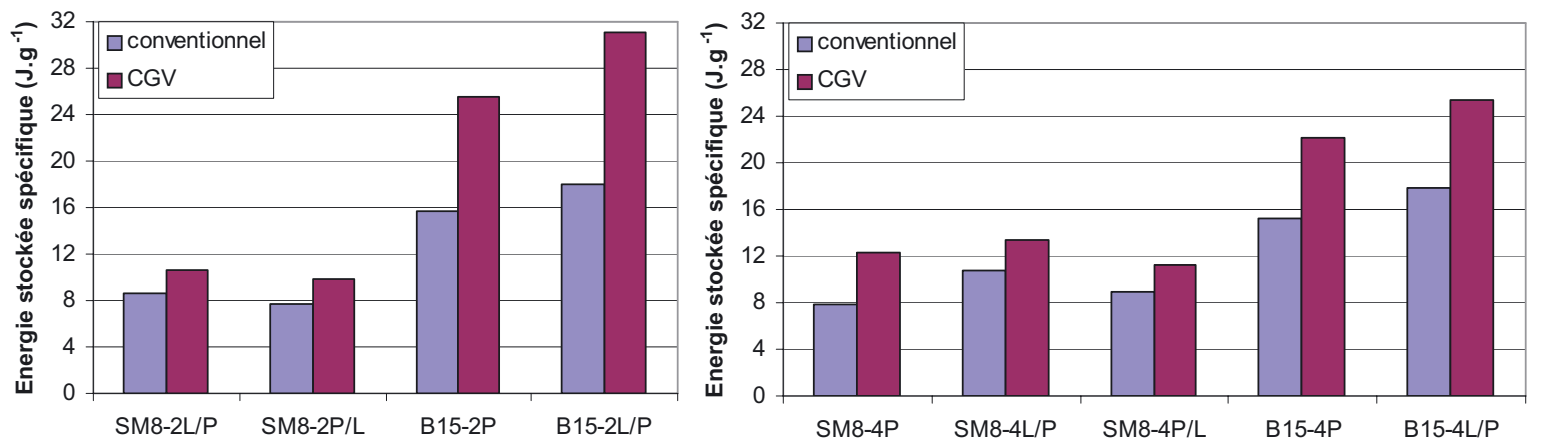

Fig. 5. Énergie stockée spécifique à $400 \mathrm{MPa}$ pour chacune des poudres et des méthodes de mise en forme.

pour chacune des poudres testées en CGV à énergie de frappe constante.

Le fait de passer de 2 à $4 \%$ en masse de phases organiques et le fait d'utiliser une poudre d'alumine plus fine diminuent la valeur du pic de contrainte principal. L'utilisation d'un plastifiant seul comme ajout organique permet l'obtention de pic de contrainte élevé et plus la quantité de liant augmente dans la phase organique, moins le pic de contrainte est important. Enfin, utiliser une poudre plus fine diminue considérablement la valeur du pic de contrainte principal.

En mise en forme CGV, toutes les poudres ayant un plastifiant seul comme ajout organique présentent des défauts rédhibitoires tels que des détachements de matière importants voire un éclatement complet après éjection. La présence d'un liant est donc nécessaire pour l'élaboration de comprimés par CGV . Les poudres avec $2 \%$ de mélange liant/plastifiant donnent des comprimés ayant un léger défaut d'écaillage surfacique, sauf ceux issus de la poudre SM8-2P/L qui sont parfois délaminés. Enfin tous les comprimés issus de poudre ayant $4 \%$ en masse d'un mélange liant/plastifiant donnent des comprimés sains.

En pressage conventionnel, toutes les poudres à l'exception de la SM8-2P ont permis l'obtention de comprimés sains. Des formulations adaptées à la mise en forme conventionnelle ne le sont pas forcément pour la CGV.

\subsection{Notion d'énergie stockée spécifique}

Les courbes de contrainte en fonction de la déformation permettent d'avoir accès à l'énergie stockée (cf. Fig. 2) par la poudre à une contrainte donnée (ici $400 \mathrm{MPa}$ ). En divisant cette énergie stockée par la masse du comprimé, on a accès à l'énergie stockée spécifique qui donne des renseignements sur le comportement de la poudre à la compression. Dans le cas des comprimés CGV, l'énergie stockée est déterminée en calculant la différence entre l'énergie reçue jusqu'à $400 \mathrm{MPa}$ et l'énergie rendue à partir de $400 \mathrm{MPa}$ afin de comparer avec le pressage conventionnel. La figure 5 regroupe les résultats obtenus en terme d'énergie stockée spécifique pour chacune des poudres et des méthodes d'élaboration.

Les résultats montrent, pour une formulation organique donnée, que plus une poudre est fine, plus il faut lui fournir d'énergie pour atteindre une contrainte désirée. Les poudres ayant un plastifiant seul comme ajout organique nécessitent le moins d'énergie, l'incorporation d'un liant rigide fait qu'il faut fournir plus d'énergie pour réorganiser la poudre.

Dans le cas de l'alumine SM8, le fait d'augmenter la quantité d'ajouts organiques provoque un accroissement de l'énergie stockée ce qui se caractérise par une plus grande difficulté de réarrangement. Ce résultat se retrouve plus difficilement dans le cas de l'alumine B15 où les forces de surface prédominent par rapport à la résistance à la compression offerte par les ajouts organiques.

Pour les comprimés CGV, il y a une concordance logique entre l'énergie stockée et le pic de pression principal : une énergie stockée spécifique importante se traduit par un pic de contrainte principal moins élevé pour une énergie d'impact donnée. Il est également constaté que les énergies stockées spécifiques en CGV sont plus importantes qu'en pressage conventionnel.

\subsection{Résistance mécanique spécifique}

En effectuant des essais de rupture diamétraux sur les échantillons élaborés conventionnellement, il est possible de connaître la résistance mécanique spécifique en divisant la contrainte de rupture à la masse volumique du comprimé [3]. Cette résistance spécifique est linéairement liée à l'énergie stockée spécifique des comprimés; en effet les calculs montrent que la conversion de l'unité $\mathrm{MPa} \cdot \mathrm{cm}^{3} \cdot \mathrm{g}^{-1}$ est équivalente à l'unité

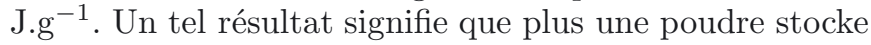
d'énergie, plus elle est difficile à comprimer, mais le comprimé obtenu est au final plus résistant et un usinage sans endommagement de la pièce peut être envisagé. Les figures 6 et 7 illustrent les résultats obtenus lors de la compression diamétrale des pièces conventionnelles en terme de résistance mécanique spécifique et la relation avec l'énergie spécifique stockée. En raison de la taille massive des comprimés CGV, les essais de rupture diamétraux n'ont pu être réalisés.

Les poudres qui ont un plastifiant seul pour ajout organique donnent les résistances les plus médiocres. L'incorporation de molécules liantes dans la phase organique 


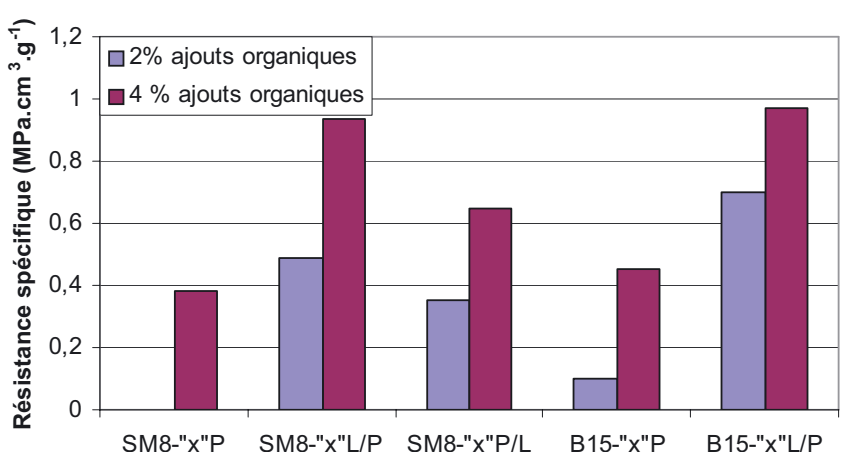

Fig. 6. Résistance mécanique spécifique des comprimés élaborés conventionnellement.

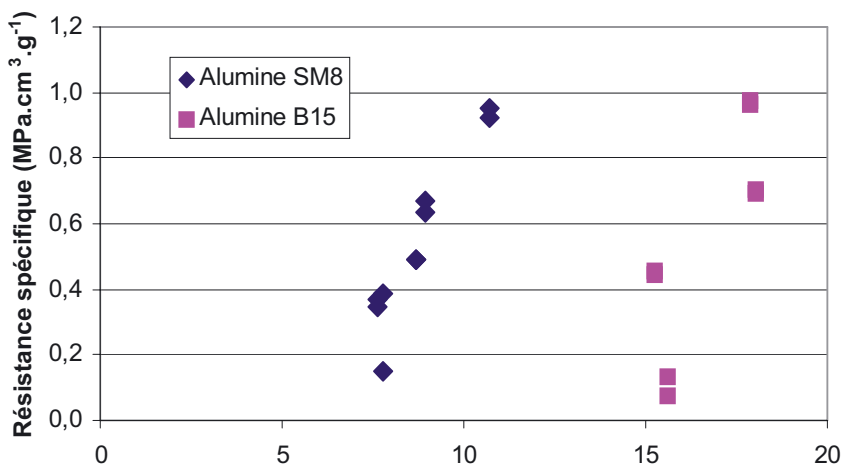

Fig. 7. Résistance mécanique spécifique des comprimés conventionnels en fonction de l'énergie stockée spécifique.

permet une augmentation de cette résistance. Une plus forte quantité d'ajouts organiques accroît la résistance mécanique des comprimés. Enfin l'affinement des cristallites favorise la tenue des comprimés pour une formulation donnée.

La relation linéaire entre la résistance mécanique spécifique et l'énergie stockée spécifique est établie. Toutefois la taille des cristallites est un paramètre à prendre en considération : une poudre fine stocke plus d'énergie au cours de la compression, mais une valeur élevée de cette énergie veut également dire que la poudre est difficile à mettre en forme.

\subsection{Comparaison de la densité des comprimés}

Après un cycle de déliantage à $600{ }^{\circ} \mathrm{C}$ afin d'éliminer toute présence de phase organique, la densité de chacun des comprimés élaborés en CGV comme en conventionnel est comparée en figure 8.

Quelle que soit la méthode de mise en forme, le fait de passer de 2 à $4 \%$ d'ajout organique provoque une diminution de la densité du comprimé. Ceci est dû à plus d'espaces vacants laissés dans le comprimé après élimination des ajouts organiques.

Les pièces ayant un plastifiant seul comme ajout ont une densité à vert plus élevée que celle ayant le mélange liant/plastifiant.
Le fait de diminuer la taille des cristallites donne, à pression de mise en forme équivalente, des comprimés de densité moins élevée; ceci est dû aux forces de surface et de friction bien plus importante.

Les comprimés CGV sont plus denses que les comprimés conventionnels : en effet, les comprimés CGV élaborés à partir d'une poudre B15 avec liant et plastifiant ont été formés à $400 \mathrm{MPa}$ tout comme les comprimés conventionnels qui ont une densité inférieure de $3 \%$ minimum. Ces densités supérieures sont certainement liées aux énergies stockées plus importantes que celles obtenues en pressage conventionnel.

\subsection{Utilisation du modèle de Lamy}

Le modèle de Lamy et al. a été utilisé pour simuler les essais de compression conventionnelle soit à partir de la porosité initiale $\left(\varepsilon_{0}\right)$ des poudres non tassées soit à partir des valeurs obtenues après précompaction à $60 \mathrm{MPa}$ et en imposant la valeur obtenue $\varepsilon_{0}$ précédemment. Dans les deux cas, le modèle donne des coefficients voisins, il est donc possible d'exploiter ces coefficients pour des poudres précomprimées suivies de compactions conventionnelles ou CGV. La porosité initiale prise en compte étant celle de la poudre non précomprimée, la concordance entre la théorie et l'expérience est effectuée à partir de $60 \mathrm{MPa}$, comme illustré dans la figure 9 avec les données issues de la compression conventionnelle et CGV de la poudre SM8-4L/P.

Le tableau 2 résume les résultats obtenus sur l'ensemble des poudres. Les coefficients $k_{\mathrm{f}}$ et $k_{\mathrm{CN}}$ ont des valeurs de l'ordre de $10^{-3} \mathrm{MPa}^{-1}$ tandis que $\psi$ est de l'ordre de $10^{-1} \mathrm{MPa}^{-1}$. Par ailleurs $\varphi$ est de l'ordre de 0,3. Les granules ne se fragmentent donc pas comme illustrés sur la figure 10 .

En considérant que $k_{\mathrm{f}}$ et $k_{\mathrm{CN}}$ sont négligeables devant $\psi$, on peut simplifier l'équation de Lamy et al. qui devient alors :

$$
\frac{\varepsilon}{1-\varepsilon}=\frac{\varepsilon_{0}}{1-\varepsilon_{0}}\left[\varphi \mathrm{e}^{-\left(k_{\mathrm{f}}+k_{\mathrm{CN}}\right) P}+(1-\varphi) \mathrm{e}^{-\psi P}\right] .
$$

Pour les poudres étudiées, le modèle de Lamy repose donc sur les coefficients $\varphi, \psi$ et la somme $k_{\mathrm{f}}+k_{\mathrm{CN}}$.

Le coefficient $\varphi$ des comprimés élaborés par CGV est plus élevé que celui de leur homologue conventionnel. Ceci pourrait être dû à des effets de paroi augmentant localement la porosité $\varepsilon_{0}$ autour des bords du récipient $(\varnothing=20 \mathrm{~mm}$ en conventionnel, $\varnothing=50 \mathrm{~mm}$ en CGV). L'augmentation de la quantité des ajouts organiques est sans grande incidence sur la valeur du coefficient.

En ce qui concerne le coefficient de réorganisation $(\psi)$, en comparant les essais conventionnels entre eux, on retrouve des résultats en accord avec ce qui a été vu précédemment : utiliser une alumine plus fine, la présence de liant dans les ajouts organiques et une quantité plus importante d'ajouts se traduit par une diminution du coefficient de réorganisation, donc il est alors plus difficile de réaliser un empilement compact. Globalement, ce coefficient est plus élevé en CGV qu'en conventionnel pour 

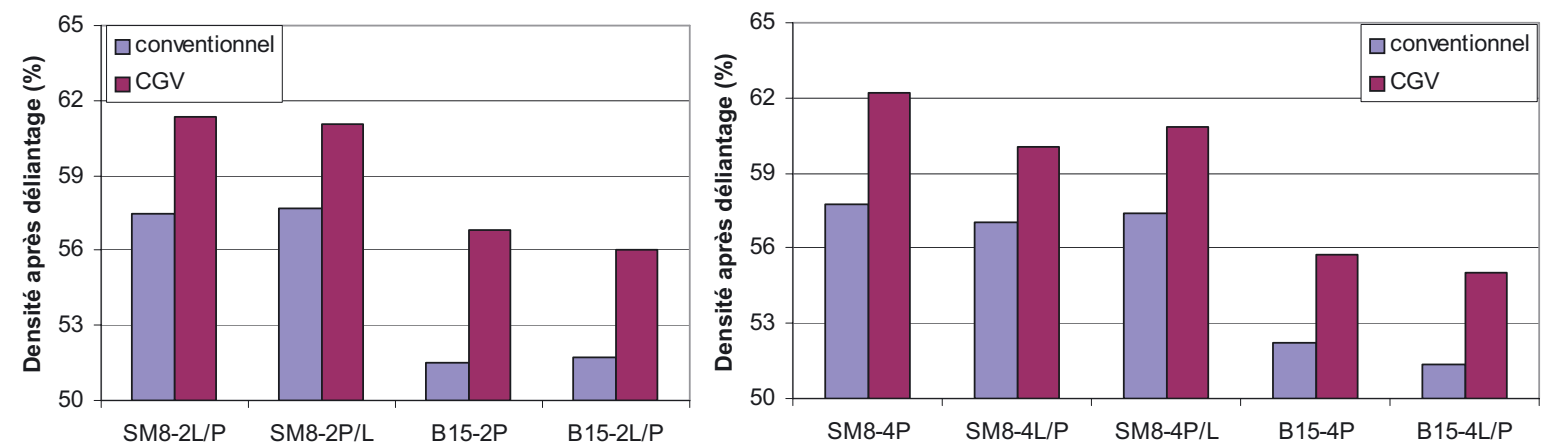

Fig. 8. Densité après déliantage des comprimés conventionnels et CGV.
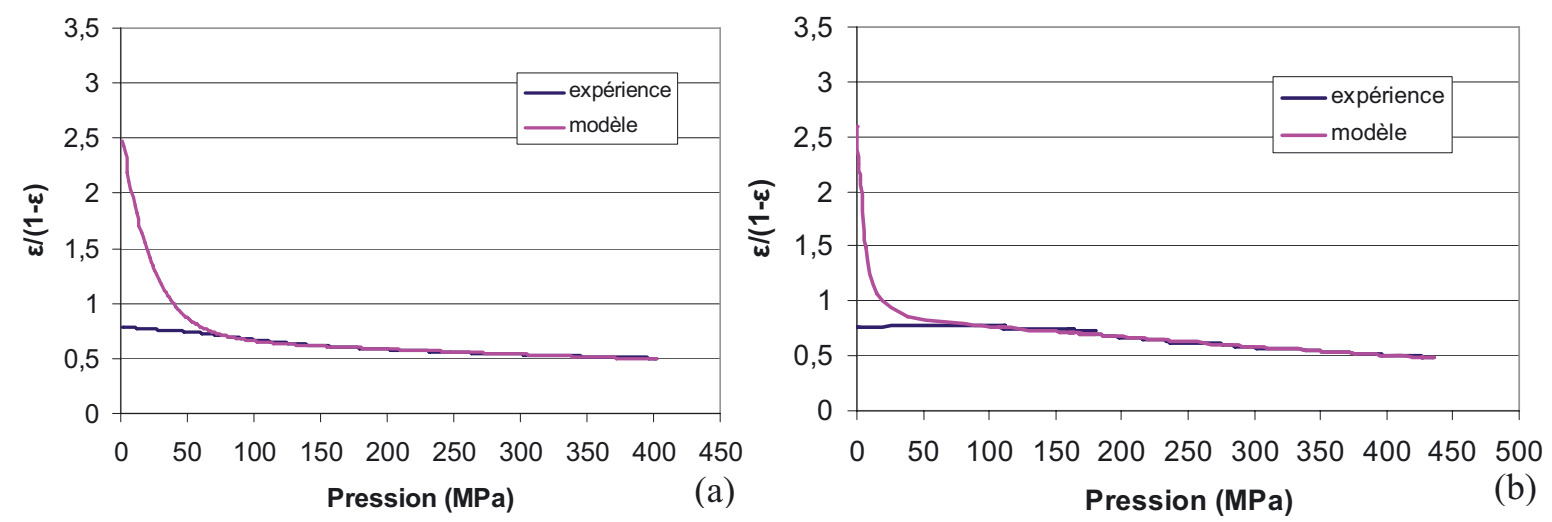

Fig. 9. Illustration de la concordance entre données expérimentale et théorique du modèle de Lamy pour la poudre SM8-4L/P comprimée, (a) conventionnellement; (b) CGV.

Tableau 2. Valeurs des coefficients de Lamy obtenus pour les différents échantillons (valeurs en $\mathrm{MPa}^{-1}$ sauf $\varphi$ sans dimension).

\begin{tabular}{|c|c|c|c|c|c|c|c|c|c|c|}
\hline & \multicolumn{5}{|c|}{ Conventionnel } & \multicolumn{5}{|c|}{$\mathrm{CGV}$} \\
\hline & SM8-2P & SM8-2L/P & SM8-2P/L & $\mathrm{B} 15-2 \mathrm{P}$ & $\mathrm{B} 15-2 \mathrm{~L} / \mathrm{P}$ & SM8-2P & SM8-2L/P & SM8-2P/L & B15-2P & $\mathrm{B} 15-2 \mathrm{~L} / \mathrm{P}$ \\
\hline$\varphi$ & - & 0,275 & 0,258 & 0,403 & 0,312 & - & 0,315 & 0,308 & 0,429 & 0,358 \\
\hline$k_{\mathrm{f}}$ & - & $6,91 \times 10^{-4}$ & $2,43 \times 10^{-7}$ & $7,41 \times 10^{-7}$ & $1,11 \times 10^{-3}$ & - & $1,01 \times 10^{-3}$ & $1,69 \times 10^{-5}$ & $1,28 \times 10^{-3}$ & $1,40 \times 10^{-3}$ \\
\hline$k_{\mathrm{CN}}$ & - & $7,68 \times 10^{-7}$ & $6,49 \times 10^{-4}$ & $9,82 \times 10^{-4}$ & $8,81 \times 10^{-7}$ & - & $5,86 \times 10^{-6}$ & $1,12 \times 10^{-3}$ & $1,00 \times 10^{-6}$ & $9,05 \times 10^{-5}$ \\
\hline \multirow[t]{2}{*}{$\psi$} & - & 0,055 & 0,062 & 0,049 & 0,047 & - & 0,042 & 0,069 & 0,067 & 0,060 \\
\hline & SM8-4P & SM8-4L/P & SM8-4P/L & B15-4P & $\mathrm{B} 15-4 \mathrm{~L} / \mathrm{P}$ & SM8-4P & SM8-4L/P & SM8-4P/L & B15-4P & $\mathrm{B} 15-4 \mathrm{~L} / \mathrm{P}$ \\
\hline$\varphi$ & 0,348 & 0,266 & 0,273 & 0,377 & 0,308 & 0,442 & 0,328 & 0,337 & 0,476 & 0,359 \\
\hline$k_{\mathrm{f}}$ & $5,43 \times 10^{-5}$ & $5,11 \times 10^{-7}$ & $8,52 \times 10^{-7}$ & $8,59 \times 10^{-4}$ & $1,09 \times 10^{-3}$ & $5,64 \times 10^{-4}$ & $3,17 \times 10^{-6}$ & $2,78 \times 10^{-5}$ & $1,00 \times 10^{-4}$ & $1,63 \times 10^{-3}$ \\
\hline$k_{\mathrm{CN}}$ & $7,12 \times 10^{-4}$ & $8,55 \times 10^{-4}$ & $8,80 \times 10^{-4}$ & $1,52 \times 10^{-4}$ & $2,77 \times 10^{-6}$ & $9,62 \times 10^{-4}$ & $1,37 \times 10^{-3}$ & $1,18 \times 10^{-3}$ & $1,75 \times 10^{-3}$ & $1,42 \times 10^{-6}$ \\
\hline$\psi$ & 0,055 & 0,045 & 0,068 & 0,037 & 0,041 & 0,189 & 0,090 & 0,121 & 0,179 & 0,125 \\
\hline
\end{tabular}

une poudre donnée. La réorganisation de la poudre se fait donc plus facilement en CGV ce qui se traduit par des empilements plus compacts. Contrairement à ce qui est observé avec les échantillons conventionnels, l'augmentation de la quantité d'ajouts organiques se traduit par une augmentation du coefficient de réorganisation. Ainsi, la CGV favorise la réorganisation d'une poudre à la condition que les ajouts organiques soient en quantité suffisante.

\section{Conclusions}

Une poudre céramique se comporte différemment à la compression suivant sa formulation organique, sa taille de grains mais également suivant la méthode de mise en forme. En pressage conventionnel, l'ajout d'une molécule liante freine la compaction mais rend le comprimé obtenu 


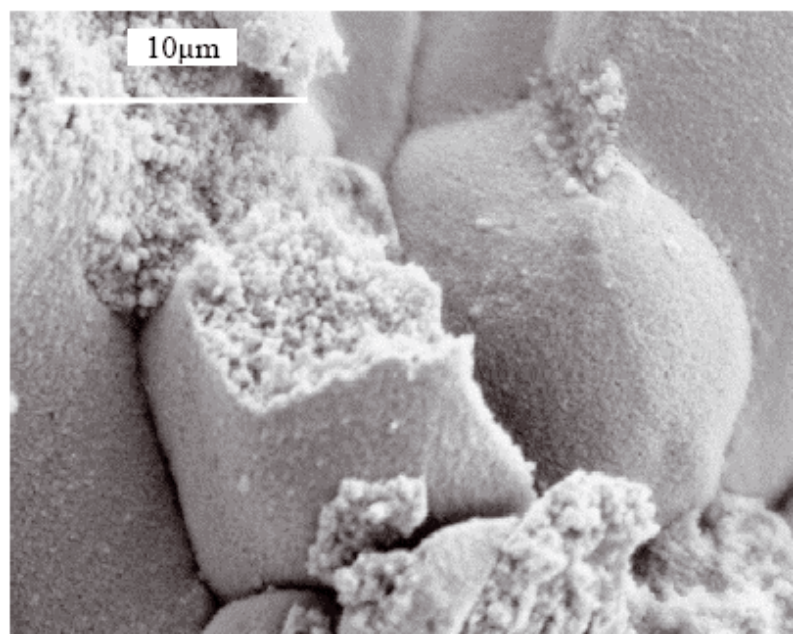

Fig. 10. Faciès de rupture d'un comprimé élaboré conventionnellement à partir de la poudre SM8-4L/P.

plus résistant; il en va de même lorsque la taille de cristallite d'une poudre est réduite.

Dans le cas d'une mise en forme par CGV, le comportement à la compression des poudres, suivant la formulation et la taille des cristallites, suit les mêmes tendances qu'en pressage conventionnel. Mais cette méthode se caractérise par une meilleure compaction des comprimés due à une meilleure réorganisation de la poudre. Cette réorganisation est d'autant meilleure que la quantité d'ajouts organiques dans la poudre est importante. La CGV permet donc l'élaboration plus rapide de pièces plus larges et plus denses et de meilleure qualité, à la condition que la formulation de la poudre soit adaptée. Il faut cependant chercher à comprendre quels mécanismes entrent en jeu en CGV afin d'expliquer la meilleure réorganisation de la poudre.

\section{Références}

[1] P. Goeuriot, A. Girinon, Compaction dynamique grande vitesse de poudres céramiques, Conséquences sur les microstructures des produits frittés, Colloque De la poudre au matériau massif, Albi, 2003

[2] P. Lamy, G. Thomas, L. Brunet, R. Erre, Modelling the porosity evolution of a powder under uniaxial compression, Propellants, Explosives, Pyrotechnics 30 (2005) 397-403

[3] J. Fell, J.M. Newton, Determination of tablet strength by diametral compression test, J. Pharmaceutical Sci. 59 (1970) 688-691

[4] D. Souriou, P. Goeuriot, O. Bonnefoy, G. Thomas, F. Doré, Compaction dynamique des poudres céramiques : conséquences sur la densification, communication matériaux, Dijon, 2006 